\title{
Germanica
}

Heiterkeit-L'allégresse au cœur de l'écriture poétique et philosophique

\section{Alexis Nouss à propos de Celan}

\section{Andrée Lerousseau}

\section{OpenEdition}

\section{Journals}

Édition électronique

URL : http://journals.openedition.org/germanica/6306

DOI : 10.4000/germanica.6306

ISSN : 2107-0784

Éditeur

Université de Lille

\section{Édition imprimée}

Date de publication : 31 décembre 2018

Pagination : 185-189

ISBN : 978-2-913857-42-1

ISSN : 0984-2632

\section{Référence électronique}

Andrée Lerousseau, « Alexis Nouss à propos de Celan », Germanica [En ligne], 63 | 2018, mis en ligne le 31 décembre 2018, consulté le 15 janvier 2021. URL : http://journals.openedition.org/germanica/6306 ; DOI : https://doi.org/10.4000/germanica.6306 


\title{
(Re)lectures
}

\section{Alexis Nouss à propos de Celan}

\author{
Andrée LEROUSSEAU \\ Université de Lille
}

L'ayant écouté lire ses poèmes, j'ai noté hâtivement :

Ni la bouche qui gouverne les mots, ni les mots dictés par les dieux sûrs. Toute sécurité retirée. Respirant par la grâce de l'irrespirable.

Jean Starobinski ${ }^{1}$

En 2010 paraissait aux éditions Le Bord de l'Eau l'essai majeur d'Alexis Nouss intitulé Paul Celan. Les lieux d'un déplacement ${ }^{2}$. S'inscrivant dans la lignée de la critique nord-américaine et à plusieurs égards dans le prolongement des analyses d'Henri Meschonnic, cette étude conserve aujourd'hui encore, en particulier pour un public français, son caractère original et novateur et sa radicalité, en ce qu'elle extrait - ou libère - l'œuvre du poète des schémas d'interprétation et des cadres de réception qui furent les siens en France, des lectures philosophiques ou esthétiques tendant à l'anhistoricité, dont le «positionnement herméneutique déhistoricisant » est qualifié par l'auteur de « regrettable stratégie d'esquive » (p. 29). L'écriture de Celan, en effet, n’a « de sens,

1. - Jean Starobinski, «L'ayant écouté lire », in Hommage à Paul Celan, Études Germaniques, 25e année, ${ }^{\circ} 3$, juillet-septembre 1970, p. 291.

2. - Nous nous proposons ici d'élargir et d'approfondir notre recension parue dans le numéro 47/2010 de Germanica.

GERMANICA, 2018, LXIII, pp. 185 à 189. 
de fonction et de mémoire que de l'après-Auschwitz » (p. 44), dans un monde interdisant désormais toute quiétude, où la conscience est d'emblée soumise à l'assombrissement et ne peut livrer son contenu qu'à partir de l'obscur ${ }^{3}$. L'essai entend également promouvoir et encourager une « lecture méridienne », une approche qui «permet de se garder d'un savoir d'autorité, d'une lecture maîtrisée et surtout maîtrisante » (p. 68) à laquelle le lecteur intimidé ou paresseux pourrait être tenté de s'en remettre : « Nulle autorité gardienne de l'interprétation », prévient l'auteur (p. 60). C'est ainsi en toute liberté, dans une écoute vigilante, qu'Alexis Nouss marche sur les traces fragiles de Celan, cheminant et tâtonnant avec lui vers ce quelque chose qui ouvre un espace à l'interprétation, alors que s'impose au lecteur le sentiment d'une étrange proximité de l'exégète avec le poète, d'une sorte de partage ou d'intimité discrète et infiniment respectueuse qu'il faudrait toutefois se garder de qualifier de complicité, terme inadéquat pour appréhender la condition du survivant commune à l'auteur et à Celan. Cette condition même implique le refus de tout confort, de toute facilité, et le rejet de tout réflexe herméneutique par le renoncement aux approches communes rendues inopérantes par l'événement («Was geschah $\left.»^{4}\right)$.

Alexis Nouss propose de traduire «Was geschah» (« Ce qui s'est passé », selon l'acception courante, au plus proche de l'allemand) par «Ce qui s'est fait », en résonnance avec « Ce qui s'est fait, est fait » ( Was geschehen ist, ist geschehen »), expression plus radicale que la formulation utilisée par Celan lui-même, mais qui traduit au plus près l'irrémédiabilité du crime perpétré (p. 265). Le paradoxe de " l'aprèsAuschwitz » est de fait qu'il n'y a pas « d'après », au sens d'une page qui serait tournée sur la chaîne du temps, comme tendrait à le laisser entendre «Ce qui s'est passé ». D'où le constat que « la seule postériorité tient dans un langage, une parole, une écriture qui disent cette impossibilité d'une postériorité chronologique » (p. 268). Il n'y a pas de rémission, pas de réparation ou de restitution possibles ${ }^{5}$. Le mot-clé qui permet d'entrer dans la poésie de Celan et de « suivre son méridien », est le « déplacement » qui innerve sa poétique (p. 87), notion autour de laquelle l'essai s'articule et se déploie, et qui contraint le commentateur à effectuer lui-même un écart, à l'instar du traducteur, l'herméneutique

3. - On songe bien sûr au recueil de Nelly Sachs, Sternverdunkelung (littéralement «L'obscurcissement de l'étoile»), paru en 1949.

4. - Titre d'un poème de Die Niemandsrose, reprenant le terme utilisé par Celan dans son « Discours de Brême » pour désigner la Shoah (cf. p. 65 à ce propos).

5. - Au-delà de la douleur partagée et de la proximité qui les lie, on mesure ainsi au fil des pages la distance qui sépare Celan de Nelly Sachs, l'amie et la sœur, celle qui, «malgré tout », conservait la foi et le fragile espoir que «peut-être » une réparation du monde au sens mystique du Tikkun Olam - fût-elle provisoire et toujours à recommencer - serait possible. 
interprétative rejoignant ainsi celle de la traduction. Quant au lecteur, il est en quelque sorte mis en condition, invité à se décentrer - à se « déporter »- grâce aux textes superbes et profonds 6 qui tiennent lieu de prélude à chaque partie et qui l'entraînent en une sorte de pèlerinage vers les lieux qui furent ceux du poète et le préparent à effectuer à son tour à la suite de Celan et de l'auteur le quadruple déplacement du sens, du temps, de la langue et de la vie.

C'est vainement que l'on chercherait une plénitude du sens dans le poème qui, contrairement à une idée reçue, n'infirme nullement, mais offre au contraire une illustration, "voire un étayage » à l'affirmation d'Adorno : « [...] écrire un poème après Auschwitz est barbare, et ce fait affecte même la connaissance qui explique pourquoi il est devenu impossible d'écrire aujourd'hui des poèmes $\gg^{7}$ (p. 34). D'où l'obligation de sortir des cadres - somme toute rassurants - offrant un relatif confort interprétatif, le « déplacement du sens » impliquant une radicalisation. Il convient ainsi de se mouvoir en direction d'une « éthique fondée sur le déséquilibre et l'incommensurabilité » (p. 324), de s'éloigner de Buber pour se rapprocher de Lévinas, plus radical en ce que son œuvre se place sous le signe de «l'in-quiétude»:

Le thème récurrent du $i c h-d u$, je et tu, dans les poèmes de Celan ne doit pas être confondu avec la pensée du dialogue chez Martin Buber ou chez Bakthine car tous deux supposent un cadre, transcendant ou immanent, qui accueille, voire suscite une dualité et une interlocution. Pour Celan, le poème se tient au bord de lui-même. (p. 315)

C'est donc avec raison que Stéphane Mosès traduit Gespräch im Gebirg par Entretien dans la montagne 8 : «Entre-tien : quelque chose se "tient entre", que l'on peut nommer silence », écrit Alexis Nous (p. 324).

Ainsi que nous l'avons déjà souligné, le temps se trouve également déplacé, dans une impossibilité de renouer avec une logique de la continuité et un refus de s'installer ou de se réfugier dans la «temporalité frauduleuse d'une histoire qui se continue » (p. 363) illustrée par la

6. - Nous citerons pour exemple ces quelques lignes, sobres et bouleversantes : « Pour un homme de cinquante ans, de taille et de corpulence moyenne, le Pont Mirabeau se parcourt approximativement en 2 minutes, quinze secondes. L'empirique contre l'émotif. Ce qui fait à peu près 240 pas. Le factuel opposé au fatal. Ce qui correspond à peu près à une centaine d'inspirations/expirations. Le détail contre le déni./ Mais saiton à quelle allure marche un homme qui va se suicider ? De toute manière, il ne va pas franchir toute la distance » (p. 347).

7. - Theodor W. Adorno, Prismes. Critique de la culture et société, trad. G. et R. Rochlitz, Paris, Payot, 1986, p. 23. Le philosophe devait, ainsi que chacun sait, revenir sur ce verdict.

8. - Paul Celan, Entretien dans la montagne, trad. Stéphane Mosès, Paris, Éd. Michel Chandaigne, 1990. 
trompeuse et fausse virginité d'une nature recouvrant ses droits, d'une végétation toute neuve dissimulant les grands charniers de l'Est ${ }^{9}$. Passionnante et particulièrement pertinente est la réflexion que propose Alexis Nouss sur le «temps d'Auschwitz », appréhendé à partir de la notion de «posthume », en référence entre autres à Jean Cayrol ${ }^{10}$ :

Le posthume, dans cette approche, perd sa définition d'événementialité postérieure à la mort, de transmission d'une essence d'un en-deçà à un au-delà de la mort (un enfant ou une œuvre posthume), son sens de résurrection en somme : le posthume n'est pas un temps rapporté, ni reporté mais déporté [...] le temps de la déportation, le temps des camps. Un temps d'une vie déplacée (p. 354).

«La syncope frappe le langage comme le temps » (p. 183), et « Ce qui s'est fait » ne peut être formulé dans une langue restituée ou rédimée: l'écart linguistique - et ce dans le travail même de Celan traducteur auquel Alexis Nouss consacre un chapitre remarquable et novateur « métaphorise un écart temporel, la déchirure de l'histoire » (p. 212). Il faut alors trouver ou inventer une langue dont la fonction n'est plus la médiation ou la communication, mais la « monstration» (p. 279), qui «montre » ce qui ne peut être figuré et échappe à la représentation, une langue mutilée, un bégaiement qui exprime et porte la fêlure, ou plutôt la transporte, obligeant à penser là également une « catégorie du posthume [...] permettant d'accueillir un événement du dire impossible auparavant » (p. 357).

Cette «poétique du posthume » qui fait retour vers la blessure, parle à partir des brisures du silence, poésie non pas de la ruine, mais d'un reste saisi et porté dans sa contemporanéité, témoigne d'une vie déplacée, d'un « déportement vers les déportés »11. Conformément à la dynamique freudienne de l'après-coup, de la Nachträglichkeit, « le témoin devient le lieu de l'avoir-lieu de l'événement que rien ne peut accueillir » et dont il est le seul garant ou, pour reprendre la terminologie de Lévinas, «l'otage », traversé, jusqu'à en être physiquement affecté, par ce « quelque chose » qui témoigne en lui et de lui (p. 301) ${ }^{12}$, son dire devenant acte d'altérité. Il s'agira dès lors pour le poète de « creuser le langage pour y crypter l'objet manquant », toujours sur le mode de

9. - Mensonge, déni, que balaye la caméra de Lanzmann et que dénoncent ses commentaires dans son chef d'œuvre, Shoah.

10. - Jean Cayrol, « De la mort à la vie », in Nuit et brouillard, Paris, Fayard, 1997, p. 58. L'auteur fait également allusion à Derrida qui, dans Spectres de Marx (Paris, Galilée, 1993, p. 42) pose l'équivalence entre « déporté » et « différé ».

11. - Cf. p. 287 où l'auteur nous invite à interpréter en ce sens l'injonction du dernier vers de Pavot et mémoire : "Compte-moi au nombre des amandes ».

12. - Il serait intéressant d'analyser ce phénomène inhérent à la condition du survivant dans l'œuvre dramatique de Nelly Sachs, jusqu'alors trop ignorée. 
la traduction qui « contient cryptiquement l'original » (p. 260 et 261) : « [...] l'original disparaît dès lors qu'il est traduit et existe disparu dans et par la traduction » (p. 256). Redéfinis à partir de la dynamique de l'inconscient qu'est l'anamnèse, le souvenir et l'oubli acquièrent un sens radicalement nouveau :

« Trouver l'oubli », au sens de « trouver qu'il y a de l'oubli » et non d'oublier [...] L'anamnèse est le dévoilement de l'oubli en tant qu'oublié, qu'il y a de l'oublié et de l'oubliable [...] les derniers recueils, en leur raréfaction scripturaire, égrènent [...] l'affirmation de l'inoubliable oublié, qui est la mémoire de l'absence (p. 147-148).

En procédant, à la suite de Celan, à ces multiples déplacements, Alexis Nouss entend mettre le lecteur lui-même en position « d'otage » qui doit à son tour traverser le sas de l'écluse, en se passant de toute lisière ou de toute école : "Il n'existe pas de communauté des lecteurs de Celan » (p. 274). Chacun doit prendre en charge l'héritage de ceux qui n'ont pas eu le temps de rédiger un testament, faire acte de passation, de traduction, et cela vaut également pour le message posthume de Celan qui a valeur d'assignation et qu'Alexis Nouss résume en ces termes : «Parce que (le poème) est trace, et non plénitude signifiante, le lecteur peut y déposer son rythme, marcher sur ses pas » (p. 143, note 2). Libéré des prescriptions et des carcans d'une interprétation qui lui serait dictée, « happé par l'extériorité qui le sollicite » (p. 304), le lecteur ne peut se dérober au poème et est placé devant sa responsabilité pour l'autre.

Tandis que nous refermons l'ouvrage après sa relecture s'imposent de nouveau à nous les paroles sur lesquelles André Spire conclut sa belle préface :

Après avoir lu ce livre, vous relirez l'œuvre de Celan avec plus de passion encore. Comme moi, vous apprécierez la force du lien qu'Alexis Nouss lie entre les lieux habités par Celan et une lecture originale et subversive d'une œuvre envoûtante (p. 17). 
